\title{
छூ \\ Intrabeam scattering studies at the Cornell Electron Storage Ring Test Accelerator
}

\author{
M. P. Ehrlichman, * W. Hartung, B. Heltsley, D. P. Peterson, N. Rider, D. Rubin, D. Sagan, J. Shanks, and S. T. Wang \\ Cornell Laboratory for Accelerator-based Sciences and Education, Cornell University, Ithaca, New York 14853-8001, USA \\ R. Campbell and R. Holtzapple \\ Department of Physics, California Polytechnic State University, San Luis Obispo, California 93407-0404, USA
}

(Received 29 July 2013; published 1 October 2013)

\begin{abstract}
Intrabeam scattering (IBS) limits the emittance and single-bunch current that can be achieved in electron or positron storage ring colliders, damping rings, and light sources. Much theoretical work on IBS exists, and while the theories have been validated in hadron and ion machines, the presence of strong damping makes IBS in lepton machines a different phenomenon. We present the results of measurements at CesrTA of IBS-dominated beams, and compare the data with theory. The beams we study have parameters typical of those specified for the next generation of wiggler-dominated storage rings: low emittance, small bunch length, and an energy of a few GeV. Our measurements are in good agreement with IBS theory, provided a tail-cut procedure is applied.
\end{abstract}

DOI: 10.1103/PhysRevSTAB.16.104401

PACS numbers: 41.75.Ht, 29.20.db, 42.72.-g, 29.27.-a

\section{INTRODUCTION}

Next-generation lepton storage rings are presently being designed for light sources, damping rings, and other applications [1-4]. These designs are intended to reach new records for high stored currents and low emittances. This will require new accelerators to operate with higher charge per bunch, more bunches per beam, and smaller bunch dimensions. Intrabeam scattering (IBS) is a single bunch, collective effect that limits the density of particle beams [5] which will likely be one of the mechanisms that limit the performance of future rings. The consequences of IBS can be interpreted as either a per-bunch current limit or a lower bound on the emittance of a bunch with a given charge. These limits depend on the optics, beam energy, radiation damping time, etc.

Intrabeam scattering has been studied in detail at $p$ and $\bar{p}$ [6-8], and heavy ion colliding-beam machines [9]. In such machines, IBS slowly dilutes the emittance of the beam and imposes a luminosity lifetime. Good agreement was found between IBS theory and experiment at the Relativistic Heavy Ion Collider (RHIC) at Brookhaven National Lab [9]. Lattices which reduce IBS growth by minimizing the dispersion invariant $\mathcal{H}_{a}=\gamma_{a} \eta_{a}^{2}+$ $2 \alpha_{a} \eta_{a} \eta_{a}^{\prime}+\beta_{a} \eta_{a}^{\prime 2}$ have been implemented at RHIC and are used regularly for colliding-beam experiments [10]. For beams of protons and antiprotons, good agreement between theory and measurements was found at the Tevatron [6].

\footnotetext{
*mpe5@cornell.edu
}

Published by the American Physical Society under the terms of the Creative Commons Attribution 3.0 License. Further distribution of this work must maintain attribution to the author(s) and the published article's title, journal citation, and DOI.
Electron and positron beams in rings reach equilibrium much more rapidly than hadron beams, hence IBS in lepton rings manifests itself differently. Lepton machines have strong radiation damping, and the equilibrium emittance is determined by a balance between radiation damping and quantum excitation. Typical damping times are on the order of tens of milliseconds. The quantized nature of IBS contributes a random motion to the scattered particles, which tends to increase the phase-space volume of the bunch. The random excitation due to IBS equilibrates with radiation damping to determine the beam size. The result is a current-dependent emittance.

Large-angle scattering events that kick particles outside the core of the bunch and contribute to particle loss and beam halo are relatively rare. Small-angle scattering events are more common. The former are commonly referred to as Touschek scattering, and the latter as intrabeam scattering. The emphasis in this paper is on intrabeam scattering.

IBS in electron beams has been studied at the Accelerator Test Facility (ATF) at KEK [11], where detailed measurements of the current dependence of the bunch energy spread and length are in good agreement with theory. Measurements of the transverse dimensions at ATF, however, are not as complete.

CesrTA is a repurposing of the Cornell Electron Storage Ring (CESR) as a test accelerator for future low-emittance storage ring designs [12]. CesrTA is a wiggler-dominated storage ring, with $90 \%$ of the synchrotron radiation produced by twelve $1.9 \mathrm{~T}$ superconducting damping wigglers. Some parameters for CesrTA are given in Table I. Design and analysis of CesrTA is done using the BMAD relativistic charged beam simulation library [13]. Measured $a$-mode (horizontal-like), single particle geometric emittance $\epsilon_{a}$ is $3.4 \mathrm{~nm} \mathrm{rad}$. The minimum measured $b$-mode (vertical-like) emittance at the time of these measurements is $\epsilon_{b} \approx 20 \mathrm{pm} \mathrm{rad}$, and arises from 
TABLE I. Machine parameters for IBS measurements.

\begin{tabular}{lc}
\hline \hline Beam energy $(\mathrm{GeV})$ & 2.085 \\
Circumference $(\mathrm{m})$ & 768 \\
rf frequency $(\mathrm{MHz})$ & 499.765 \\
Horizontal tune $\left(Q_{x}\right)$ & 14.624 \\
Vertical tune $\left(Q_{y}\right)$ & 9.590 \\
Synchrotron tune $\left(Q_{z}\right)$ & -0.065 \\
Transverse damping time $(\mathrm{ms})$ & 56.6 \\
\hline \hline
\end{tabular}

sources such as magnet alignment, field errors, and quality of beam-based optics corrections. Subsequent machine studies have reduced the $b$-mode emittance by another $50 \%$, at which point the $b$-mode emittance is dominated by sources unaffected by optics correction [14]. The flexibility of the CesrTA optics allows precise control of $b$-mode emittance above that minimum. We are able to vary $b$-mode emittance by using closed coupling bumps to introduce a localized vertical dispersion in the damping wigglers. In this way, vertical emittance can be increased by an order of magnitude without affecting the global optics. The bunch length is determined by the $\mathrm{rf}$ accelerating voltage. With a voltage of $6 \mathrm{MV}$, the bunch length is about $10.5 \mathrm{~mm}$. Measurements were made with bunch charges ranging from $1.6 \times 10^{9}$ to $1.6 \times 10^{11}$ particles/bunch (0.1 to $\left.10 \mathrm{~mA}\right)$.

CesrTA is instrumented for precision bunch size measurements in all three dimensions. Vertical beam size measurements are made using an $\mathrm{x}$-ray beam size monitor (xBSM), which images $x$ rays from a hard bend magnet through a pinhole onto a vertical diode detector array $[15,16]$. The instrument images the beam turn by turn, allowing bunch position and size measurement on each bunch passage. These turn-by-turn images can be analyzed collectively to reveal beam motion and beam size fluctuations. The images can also be summed over all turns to improve average beam size accuracy at low current, after correcting for beam motion. Horizontal beam size measurements are made with a visible-light interferometer [17]. The interferometer is used to image visible synchrotron radiation on a charge-coupled device (CCD) that is exposed for about 400 turns at high current or about 40000 turns at low current. Bunch length measurements are done with a streak camera using visible light from a bending magnet [18]. The horizontal, vertical, and longitudinal data plotted in this paper are the binned average over measurements within a current range. The error bars are the statistical uncertainty of the measurements within the bin.

Validation of the beam size instrumentation includes checking for intensity dependent systematics using filters, and size systematics by varying source-point betatron functions. The horizontal beam size monitor also undergoes direct calibration with a source of known size [17].

One of the goals of the CesrTA IBS investigation is to improve on the ATF results by including detailed measurements of the bunch charge dependence of the transverse beam sizes. In addition to robust instrumentation, CesrTA has independently powered quadrupoles and the capability to store larger single-bunch charges. This flexibility allows for measurements at CesrTA in a greater variety of conditions.

We use the IBS formalism developed by Kubo and Oide [19] to describe the data. The formalism is a generalization of the Bjorken-Mtingwa description [20] and uses an eigen-decomposition of the beam $\Sigma$ matrix rather than the traditional Twiss parameters. This formalism naturally handles arbitrary coupling among the three beam dimensions.

In this paper, we describe the CesrTA IBS experiments, and compare the results to both analytic theory and Monte Carlo simulations. Some of the results shown here were first presented at the 2012 International Particle Accelerator Conference [21]. The present paper provides a more complete description and theoretical framework for the results. Further details can be found in [22].

\section{THEORY}

The IBS formalism outlined here is described succinctly by Kubo [23] and in detail by Kubo and Oide [19]. It is based on changes to the second-order moments of the $\mathbf{\Sigma}$ matrix of the beam distribution in the frame of the bunch

$$
\Delta\left\langle\bar{p}_{i} \bar{p}_{j}\right\rangle=c_{I} \mathbf{R}\left\langle\delta \mathbf{w}^{2}\right\rangle \mathbf{R}^{T},
$$

where $\mathbf{R}$ is a matrix of eigenvectors defined below, $c_{I}$ is proportional to the bunch charge, and

$$
\left\langle\delta \mathbf{w}^{2}\right\rangle=\left(\begin{array}{ccc}
\left\langle\delta w_{1}^{2}\right\rangle & 0 & 0 \\
0 & \left\langle\delta w_{2}^{2}\right\rangle & 0 \\
0 & 0 & \left\langle\delta w_{3}^{2}\right\rangle
\end{array}\right),
$$

$\left\langle\delta w_{1}^{2}\right\rangle,\left\langle\delta w_{2}^{2}\right\rangle$, and $\left\langle\delta w_{3}^{2}\right\rangle$ are the rates of change of the normal mode 2 nd order moments.

IBS refers to scattering among nearby particles. The 2 nd order moments of the $\mathbf{\Sigma}$ matrix describe the momentum spread of the entire bunch. What is needed is the "local" momentum spread, or the spread in the momentum of particles inside a small spatial element of the bunch. The difference between the $\boldsymbol{\Sigma}$ matrix 2nd order moments and the local moments is depicted in Fig. 1. The local momentum spread is obtained via

$$
\boldsymbol{\Sigma}_{l p p} \equiv\left\langle\bar{p}_{l i} \bar{p}_{l j}\right\rangle=\boldsymbol{\Sigma}_{p p}-\boldsymbol{\Sigma}_{x p}^{T} \boldsymbol{\Sigma}_{x x}^{-1} \boldsymbol{\Sigma}_{x p},
$$

where $\boldsymbol{\Sigma}_{p p} \equiv\left\langle\bar{p}_{i} \bar{p}_{j}\right\rangle, \boldsymbol{\Sigma}_{x x} \equiv\left\langle\bar{x}_{i} \bar{x}_{j}\right\rangle$, and $\boldsymbol{\Sigma}_{x p} \equiv\left\langle\bar{x}_{i} \bar{p}_{j}\right\rangle$.

$\boldsymbol{\Sigma}_{l p p}$ is symmetric and positive definite and can be decomposed as

$$
\boldsymbol{\Sigma}_{l p p}=\mathbf{R G R}^{T}
$$

where $\mathbf{G}$ is a diagonal matrix of the eigenvalues of $\boldsymbol{\Sigma}_{l p p}$ and the columns of $\mathbf{R}$ are the eigenvectors. The eigenvalues are denoted $u_{1}, u_{2}, u_{3}$. Note that $\mathbf{R}^{T}=\mathbf{R}^{-1}$. 


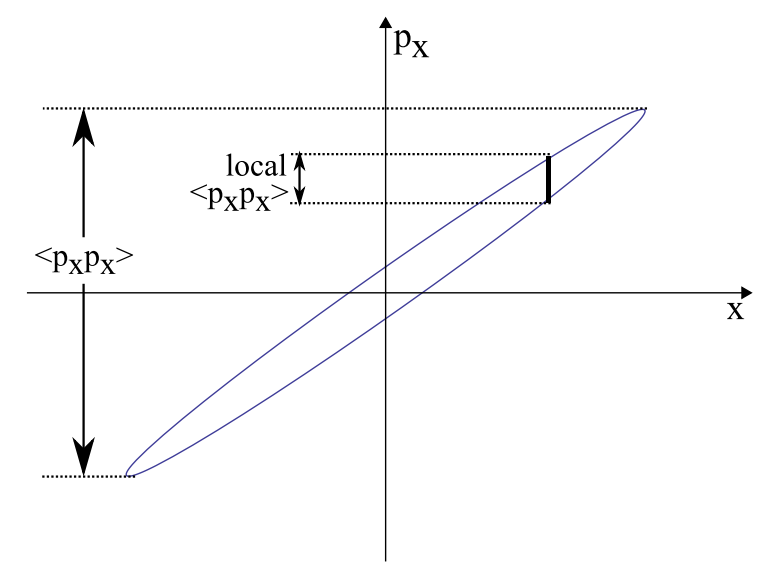

FIG. 1. The local momentum $\boldsymbol{\Sigma}$ matrix describes the distribution of momentum in a small spatial element of the bunch.

$\left\langle\delta \mathbf{w}^{2}\right\rangle$ is obtained from

$$
\begin{aligned}
& \left\langle\delta w_{1}^{2}\right\rangle=g_{2}+g_{3}-2 g_{1}, \\
& \left\langle\delta w_{2}^{2}\right\rangle=g_{1}+g_{3}-2 g_{2}, \\
& \left\langle\delta w_{3}^{2}\right\rangle=g_{1}+g_{2}-2 g_{3},
\end{aligned}
$$

where

$$
\begin{aligned}
& g_{1}=g\left(u_{1}, u_{2}, u_{3}\right), \\
& g_{2}=g\left(u_{2}, u_{1}, u_{3}\right), \\
& g_{3}=g\left(u_{3}, u_{1}, u_{2}\right),
\end{aligned}
$$

and

$g(a, b, c)=\int_{0}^{\pi / 2} \frac{2 a \sin ^{2} s \cos s}{\sqrt{\left(\sin ^{2} s+\frac{a}{b} \cos ^{2} s\right)\left(\sin ^{2} s+\frac{a}{c} \cos ^{2} s\right)}} d s$.

$g_{1}, g_{2}$, and $g_{3}$ are analogous to the temperatures of the three normal modes of the bunch.

$c_{I}$ is defined as

$$
c_{I}=\frac{r_{e}^{2} N_{e} \Delta s}{4 \pi \gamma^{4} \epsilon_{a} \epsilon_{b} \epsilon_{c}} C_{\Lambda}
$$

where $\epsilon_{a}, \epsilon_{b}$, and $\epsilon_{c}$ are the normal mode emittances of the beam, and the Coulomb $\log$ arithm $C_{\Lambda}$ will be defined in the next section. $N_{e}$ is the number of particles in the bunch, $r_{e}$ is the classical electron radius, $\gamma$ is the relativistic factor, and $\Delta s$ is the length of the region over which particles interact.

\section{A. Coulomb logarithm}

The Coulomb $\log , C_{\Lambda}$, appears in the integration of the Rutherford scattering cross section over all scattering angles. The integral diverges for small scattering angles, which correspond to large impact parameters. This requires the introduction of a largest impact parameter cutoff. We follow the prescription by Kubo and Oide [19] and use the smaller of the mean interparticle distance and the smallest beam dimension as the maximum impact parameter,

$$
b_{\max }=\min \left(n^{-1 / 3}, \sigma_{x}, \sigma_{y}, \gamma \sigma_{z}\right),
$$

where $n$ is the particle density in the bunch frame,

$$
n=\frac{N_{e}}{(4 \pi)^{3 / 2} \sigma_{x} \sigma_{y} \gamma \sigma_{z}} .
$$

As for the largest scattering angle (smallest impact parameter), both Piwinski and Bjorken-Mtingwa assume that $\theta_{\max }=\pi / 2$. It was suggested in [24] that scattering events which occur less frequently than once per particle per radiation damping time should be excluded from the calculation of the IBS rise time. This is because such events do not occur frequently enough for the central limit theorem to apply and therefore do not contribute to the Gaussian core of the beam. Such infrequent events will generate non-Gaussian tails. It is the size of the Gaussian core that we can measure, so for comparison with the data, we exclude contributions to the tails.

The canonical momentum of a particle in an electron/ positron storage ring is the sum over a history of momentum kicks that occur whenever the particle radiates a photon. The photon carries away some transverse momentum, but the emission event can also increase the transverse momentum of the particle if the photon is emitted in a region of finite dispersion. The overall effect of the emission event on the particle's momentum depends on the action, angle, and local optics where a photon is emitted. Because photon emission is stochastic and occurs at random points along the particle's trajectory, the kicks are also stochastic. According to the central limit theorem, the momenta of particles in a bunch will be normally distributed in the absence of IBS. In the presence of IBS, the distribution consists of a core which is close to Gaussian, along with non-Gaussian tails.

The amount of transverse momentum taken away by the radiated photon tends to be larger for particles with a larger transverse momentum. This leads to damping and results in an equilibrium distribution of momenta, rather than unbounded momentum diffusion. Perturbations to particle motion damp exponentially with a characteristic radiation damping time. Within one damping time, a large number of stochastic photon emission events occur. For CesrTA, about $20 \times 10^{6}$ photons are emitted per particle per damping time.

Similarly, there are a large number of small-angle intrabeam scattering events that likewise excite oscillations. These IBS events increase the width of the momentum distribution. However, very few large-angle scattering events occur per damping time. 
A particle with velocity $v$, traveling through a gas with density $\rho$, and an interaction cross section $\sigma$, will undergo scattering events at a rate $1 / \tau=\rho v \sigma$. Writing $\sigma=\pi b^{2}$, where $b$ is the effective impact parameter yields

$$
\frac{1}{\tau}=\pi \rho v b^{2} .
$$

For nonrelativistic Coulomb scattering, the impact parameter is related to the scattering angle $\psi$ by

$$
b=\frac{r_{e}}{2 \bar{\beta}^{2}} \cot \frac{\psi}{2},
$$

where $\bar{\beta} c$ is the velocity of the particles in their center-ofmomentum frame. Substituting Eq. (16) into (15) gives the rate in the lab frame at which particles are scattered into angles less than or equal to $\psi$ :

$$
\frac{1}{\tau}=\frac{1}{\gamma} \frac{\pi \rho c r_{e}^{2}}{4 \gamma^{3}\left(\epsilon \gamma_{a}\right)^{\frac{3}{2}}} \cot ^{2} \frac{\psi}{2}
$$

where $\gamma \sqrt{\epsilon \gamma_{a}}$ has been used for $\bar{\beta}, \epsilon$ is the geometric emittance, and $\gamma_{a}$ is the $a$-mode Twiss $\gamma$. The relevant beam parameters for CesrTA are shown in Table II. The rate of scattering events, $\Gamma_{s}$, in units of radiation damping time, $\Gamma_{r}$, as a function of maximum scattering angle is shown in Fig. 2(a). The tail cut excludes those events which occur less than once per radiation damping period. A measure of the sensitivity to the cutoff is illustrated in Fig. 2(b). The calculated equilibrium beam size is shown for a range of 2 orders of magnitude of the cutoff. The data shown is the same as plotted in Fig. 9(a).

The tail cut consists of restricting the calculation of the IBS growth rate to include only those events which occur at least once per particle per damping period. Events which occur less frequently than once per damping period generate lightly populated non-Gaussian tails that do not contribute to the Gaussian core. It is the Gaussian core that we can measure and that is important when determining the brightness of a light source or luminosity in a collision experiment.

The tail cut is applied by setting the minimum impact parameter as

$$
b_{\min }=\sqrt{\frac{1}{n \pi \tau_{b} \nu}}
$$

where $\tau_{b}$ is the longest damping time in the bunch frame and $\nu$ is the average particle velocity in the bunch frame. If $\epsilon_{a}$ is greater than $\epsilon_{b}$ and $\frac{\sigma_{p} \sigma_{z}}{\gamma^{2}}$, then $\nu \approx c \gamma \sqrt{\frac{\epsilon_{a}}{\beta_{a}}}$.

TABLE II. Nominal conditions for a bunch with $6.4 \times 10^{10}$ particles.

\begin{tabular}{lc}
\hline \hline Beam energy $\gamma$ & 4080 \\
Average density $\rho$ & $4.2 \times 10^{21} \mathrm{part} / \mathrm{m}^{3}$ \\
Twiss $\gamma_{x}$ & $0.51 \mathrm{~m}^{-1}$ \\
Emittance $\epsilon_{a}$ & $3.0 \mathrm{~nm} \mathrm{rad}$ \\
\hline \hline
\end{tabular}
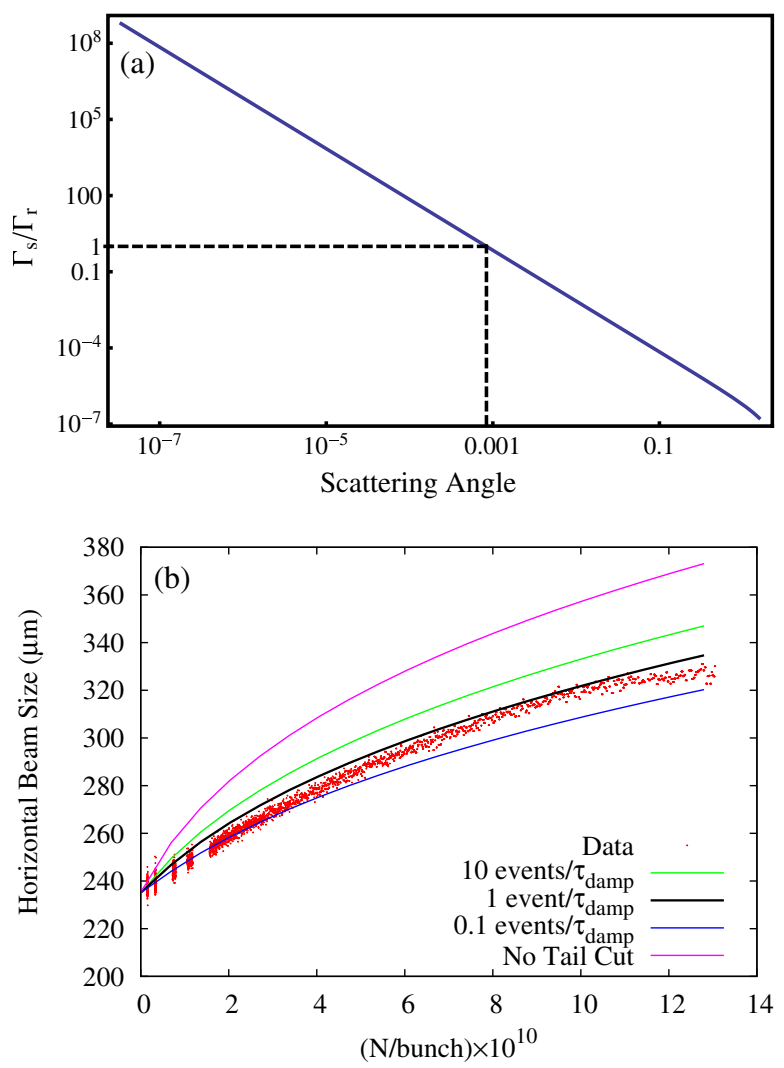

FIG. 2. (a) Events which occur less than once per damping time are excluded from the calculated growth rate. (b) Equilibrium beam size calculations assuming different cutoffs.

The computed IBS growth rate is directly proportional to the Coulomb log and is expressed as the logarithm of the maximum impact parameter over the minimum,

$$
C_{\Lambda}=\log \frac{b_{\max }}{b_{\min }} .
$$

In hadron and ion machines, such as the Tevatron and RHIC, the damping time is very long and there are enough of even the very large-angle scatters to populate a Gaussian distribution. A tail cut does not significantly affect the calculated IBS distributions for those machines. However, for machines with strong damping, such as lepton storage rings, very few large-angle scattering events occur per damping time, and applying the tail cut is essential to reliably computing the equilibrium distribution of the Gaussian core of the bunch. In CesrTA, applying the tail cut significantly changes the calculated growth rate. With the tail cut, the average Coulomb log in CesrTA at $1.6 \times 10^{10}$ particles/bunch is 9.4 . Without the tail cut, that is, if we assume that the maximum scattering angle is $90^{\circ}$, the average Coulomb log is 17.6.

\section{B. Monte Carlo IBS simulations}

In addition to the analytic IBS calculations discussed above, we have developed a Monte Carlo simulation based 
on Takizuka and Abe's plasma collision model [25]. An ensemble of 2000 particles representing the bunch distribution is tracked element by element using the BMAD standard tracking methods [13]. We use an analytic model of the damping wiggler field, which is based on a fit to a finite element calculation [26]. Tracking through wigglers is by symplectic integration.

At each element, the ensemble is converted from canonical to spatial coordinates and boosted into its center-of-momentum frame where the particles are nonrelativistic. Then Takizuka and Abe's collision model is applied: (i) The bunch is divided into cells. This enforces locality. (ii) Particles in each cell are paired off. Each particle undergoes only one collision. (iii) The change in the momentum of the pair is calculated, taking into account their relative velocities and the density of particles in the cell. The ensemble is then boosted back to the lab frame and transformed back into canonical coordinates.

Note that this is not a Monte Carlo simulation of individual scattering events. Such a simulation would require the calculation of $\frac{N !}{2}$ scattering events per element and is not computationally feasible. Takizuka and Abe's formalism calculates the expectation value of the change in the momentum of a test particle traveling through a "wind" of nearby particles. The relative velocity of the paired particles determines the velocity vector of the wind. The rate of change of the particle momentum due to scattering events is assumed to be constant through the length of the element.

A log term corresponding to the Coulomb log appears in Takizuka and Abe's formalism. The calculation of the expectation value of the change in the momentum of the particles assumes many small-angle scattering events. This method of Monte Carlo simulation is subject to the central limit theorem and tail cut in the same way as the analytic calculations.

\section{Potential well distortion (PWD)}

The bunch interacts with structures in the vacuum system, resulting in wake fields that act back on the bunch. One consequence of this is a voltage gradient along the length of the bunch. Particles at the head of the bunch lose energy to the vacuum system. Part of this energy is reflected back to the tail of the bunch, effectively transferring energy from the head of the bunch to the tail. In machines that operate above transition, particles with less energy move ahead relative to the reference particle, and those with more energy move back. The result is bunch lengthening. The amount of lengthening is sensitive to the total bunch charge, but not to the transverse dimensions of the bunch.

Energy that is reflected back into the bunch does not change the total energy of the bunch and is referred to as the inductive $(L)$ or capacitive $(C)$ part of the impedance. Energy absorbed by the vacuum system does change the total energy of the bunch and is referred to as the resistive part of the impedance $(R)$.
In the general case, the impedance is frequency dependent. Here, the effect of potential well distortion is approximated as a current-dependent rf voltage. The effective rf voltage is [27]

$$
V(\tau)=V_{\mathrm{rf}} \cos (\omega \tau+\phi)+R I_{b}(\tau)+L \frac{d I_{b}(\tau)}{d \tau},
$$

where $\tau$ is relative to the bunch center. The resistive impedance $R$ tends to shift the synchronous phase but does not contribute to lengthening. The inductive part $L$ changes the Gaussian profile of the bunch, leading to real bunch lengthening.

In principle, there is also a capacitive part to the impedance. Its effect is to shorten the bunch. In CesrTA, only bunch lengthening is observed. This is because the inductive term in the overall impedance is much larger than the capacitive. Hence, the reactive part of the impedance is modeled as entirely inductive. In theory, the inductive, capacitive, and resistive parts of the impedance could each be determined from the shape of the longitudinal profile of the bunch. However, our measurements are not detailed enough to determine if a capacitive part of the impedance is counteracting the inductive part.

A derivation of PWD based on Vlassov theory results in a differential equation for the longitudinal profile of the bunch [27],

$$
\frac{\partial \psi}{\partial \tau}=-\frac{e E_{0} \psi}{\sigma_{E}^{2} \alpha T_{0}}\left(\frac{V_{\mathrm{rf}} \cos (\omega \tau+\phi)+Q R \psi-U_{0}}{1+\frac{e E_{0} Q L \psi}{\sigma_{E}^{2} \alpha T_{0}}}\right),
$$

where $E_{0}$ is the beam energy, $\sigma_{E}$ is energy spread, $\alpha$ is momentum compaction, $T_{0}$ is the period of the ring, $V_{\mathrm{rf}}$ is the total $\mathrm{rf}$ cavity voltage, $\omega$ is the rf frequency, $\phi$ is the phase of the reference particle with respect to the $\mathrm{rf}, Q$ is the bunch charge, $U_{0}$ is the energy lost per particle per turn, $R$ is the resistive part of the longitudinal impedance, and $L$ is the inductive part of the longitudinal impedance. $\psi(\tau)$ is the longitudinal profile of the bunch. Equation (21) is used to compute the effect of various resistive and inductive impedances on the longitudinal profile of the bunch. The results are shown in Fig. 3.

We have incorporated the effect of PWD in our analytic model of IBS. Equation (21) is used to compute bunch length, including the energy spread resulting from intrabeam scattering. Comparing the measured bunch length versus current data to the simulation result, $L$ is determined to be $25.9_{-17.2}^{+18.7} \mathrm{nH}$ for positrons and $21.1_{-14.5}^{+15.3} \mathrm{nH}$ for electrons. Our bunch length predictions are largely insensitive to $R$, and we use the published value of $1523 \Omega$ given by Holtzapple et al. [18]. At the time of this writing, PWD has not been implemented in the Monte Carlo simulation.

As shown in Fig. 3, resistive impedance has a negligible effect on the length of the longitudinal profile, whereas the inductive impedance $L$ distorts the Gaussian profile and 

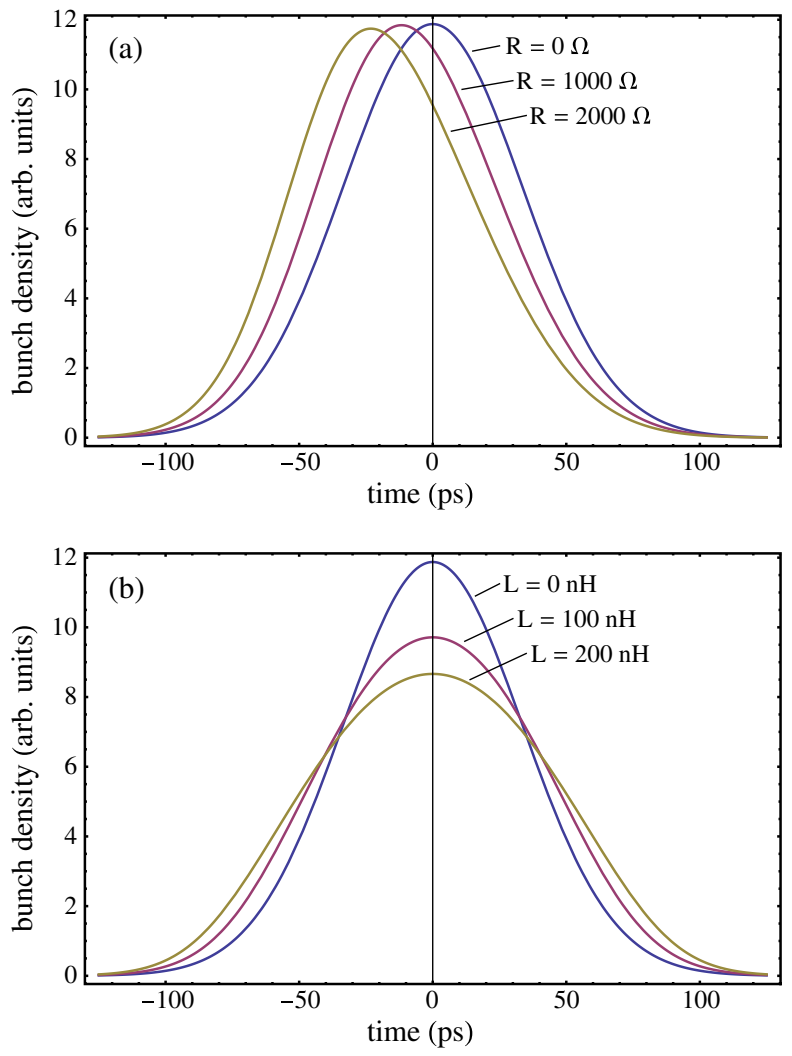

FIG. 3. Effect of (b) inductive and (a) resistive parts of the longitudinal impedance on the longitudinal profile of the bunch.

generates bunch lengthening. Figure 4 shows the contribution of the potential well distortion to the bunch length assuming various values for the inductive impedance.

The current-dependent energy spread in CesrTA is determined by measuring the dependence of the horizontal beam size on the horizontal dispersion at the instrument source point. The dispersion is varied with the help of a closed dispersion bump around the source point. The horizontal beam size is measured under two sets of conditions

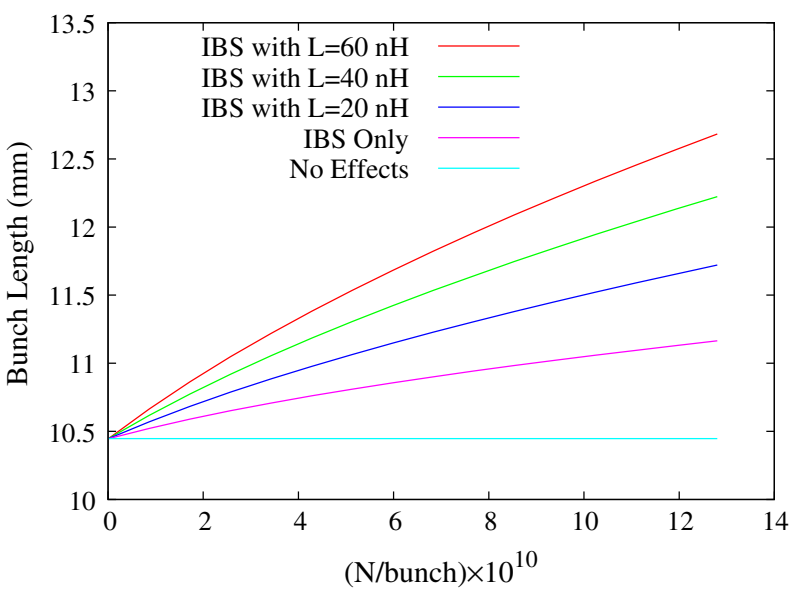

FIG. 4. Simulated effect on bunch length of PWD in combination with IBS. as the number of particles in a single bunch decays from $1.3 \times 10^{11}$ down to $2.4 \times 10^{10}$. Horizontal dispersion is $2.28 \mathrm{~cm}$ in the first set of conditions, and $22.1 \mathrm{~cm}$ in the second. The measured energy spread is $\sigma_{E} / E=(8.505 \pm$ $0.314) \times 10^{-4}$ and is independent of current within the measurement uncertainty. The design value of the fractional energy spread as determined using the standard radiation integrals is $8.129 \times 10^{-4}$. There is no evidence
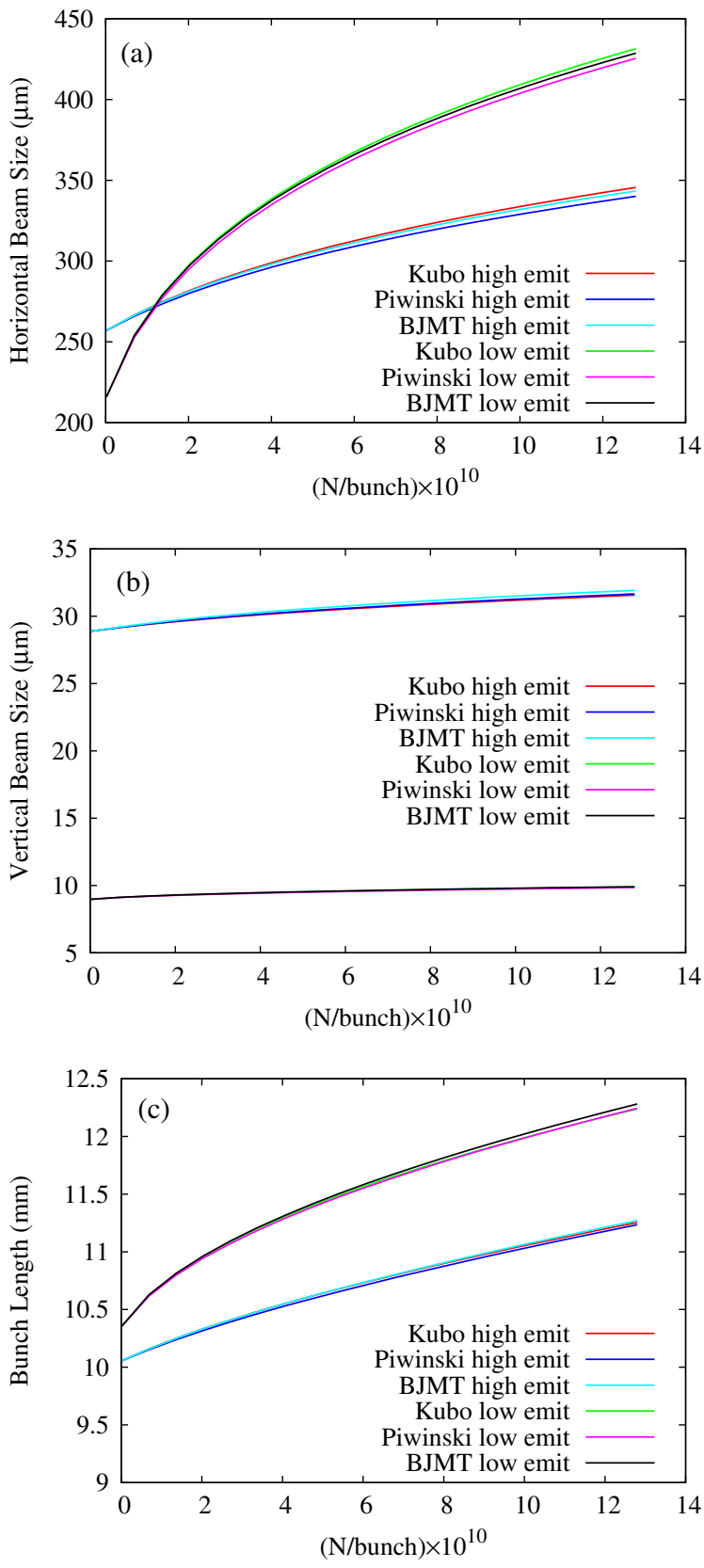

FIG. 5. Comparing (a) horizontal, (b) vertical, and (c) longitudinal beam size versus current for Kubo's, Piwinski's, and the Bjorken-Mtingwa (BJMT) IBS formalisms. The high emittance lattice has $\epsilon_{x 0}=4.6 \mathrm{~nm} \cdot \mathrm{rad}, \epsilon_{y 0}=14.3 \mathrm{pm} \cdot \mathrm{rad}$, and $\sigma_{z 0}=10.0 \mathrm{~mm}$. The low-emittance lattice has $\epsilon_{x 0}=$ $2.8 \mathrm{~nm} \cdot \mathrm{rad}, \epsilon_{y 0}=1.5 \mathrm{pm} \cdot \mathrm{rad}$, and $\sigma_{z 0}=10.3 \mathrm{~mm}$. 
of a microwave instability, which would appear as an energy spread that increases with current above some threshold current.

\section{Projected beam sizes}

Beam sizes from the simulations are obtained from the $\langle x x\rangle,\langle y y\rangle$, and $\langle z z\rangle$ elements of the $6 \times 6$ beam envelope matrix. These are evaluated at the instrumentation source points. The beam sizes obtained by this method are the projections of the beam into the horizontal, vertical, and longitudinal dimensions and are the bunch profiles actually seen by the instrumentation. This method naturally takes into account arbitrary coupling among the six normal mode phase-space coordinates of the bunch.

\section{E. Method comparison}

In addition to Kubo and Oide's method, two other commonly used methods for calculating IBS growth rates are one by Bjorken and Mtingwa [20] and a version of Piwinski's original derivation that includes derivatives of the lattice optics [28]. Figure 5 shows the equilibrium beam sizes versus current calculated using each of the three methods.

We treat the Coulomb log the same way in each method and apply the tail cut. Applying the tail cut to Piwinski's original method requires modifying the derivation so that the minimum and maximum scattering angles can be set as parameters.

Bjorken and Mtingwa's and Piwinski's methods are based on Twiss parameters. We use normal mode Twiss parameters in place of horizontal, vertical, and longitudinal Twiss parameters when evaluating either formalism. The growth rates given by the formulas are applied to the normal mode emittances.

These calculations suggest that, provided the Coulomb $\log$ is treated the same, the three most general IBS formalisms predict similar equilibrium beam sizes. For the studies shown here, transverse coupling is not strong enough to significantly impact the IBS growth rates.

\section{CURRENT-DEPENDENT TUNE SHIFT}

A current-dependent shift of the coherent tune is observed in CesrTA. At $2.1 \mathrm{GeV}$, the vertical shift was measured to be $-0.505 \pm 0.006 \mathrm{kHz} / \mathrm{mA}$. The horizontal shift was measured to be $-0.072 \pm 0.006 \mathrm{kHz} / \mathrm{mA}$. (1 kHz corresponds to a change in fractional tune of 0.0026.) The synchrotron tune has been measured versus current, and no shift was observed. These tune shifts are relevant to IBS studies because the beam size will in general depend on proximity of the coherent tune to resonance lines in the tune plane. Preparation for IBS studies includes identifying a region of the tune plane where the effect of resonance lines is minimized for the range of currents to be explored. The tune plane is scanned with direct measurement as well as tracking simulation. The experimental tune scans are performed by recording the beam sizes as the tune is varied by adjusting quadrupole strengths.

Figure 6 shows the measured dependence of vertical and horizontal coherent tunes on bunch current. The betatron frequencies are measured via a pair of spectrum analyzers connected to beam position monitor (BPM) buttons.

Figure 7 shows a simulated tune scan. The color scale shows the rms value of the vertical-like normal mode action $J_{b}$ of a particle tracked for 2000 turns, normalized by its initial value $J_{b 0}$. The thin lines are analytic calculations of the form $r Q_{x}+s Q_{y}+t Q_{z}=n$. The labels are of the form $(r, s, t, n)$. Amplitude-dependent tune shift causes the resonance lines in the simulation to be offset from the analytic calculations. The initial action $J_{b 0}$ of the tracked particle is set to be about 10 times the equilibrium emittance. The yellow line shows the range of coherent tune spanned as a bunch decays from $1.3 \times 10^{11}$ particles to $1.6 \times 10^{9}$ particles. The upper right-hand point is the zero current tune.

The simulated and experimental tune scans are generally only in approximate agreement. The lower order resonances, such as $(1,-1,-1,0)$, tend to be much broader in the experimental tune scan. The higher order resonances
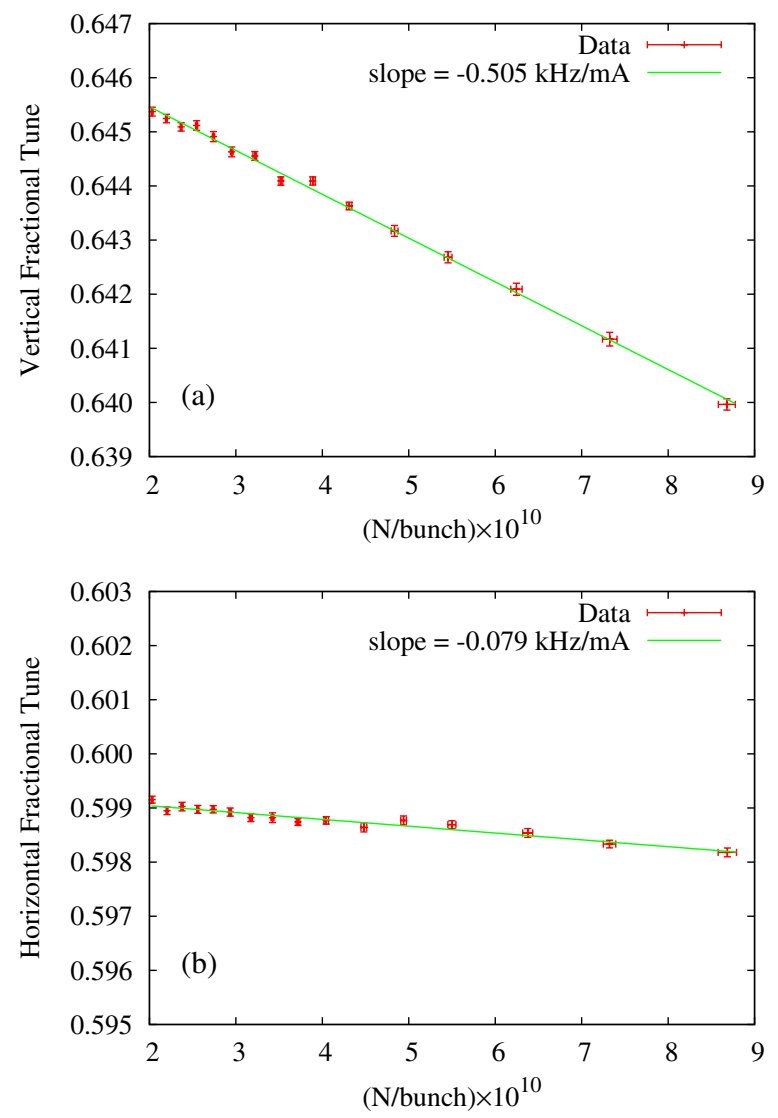

FIG. 6. Fractional coherent tune versus current for (a) the vertical and (b) the horizontal plane. The resolution of the measurement is $10^{-4}$. The revolution frequency is $390.1 \mathrm{kHz}$. 


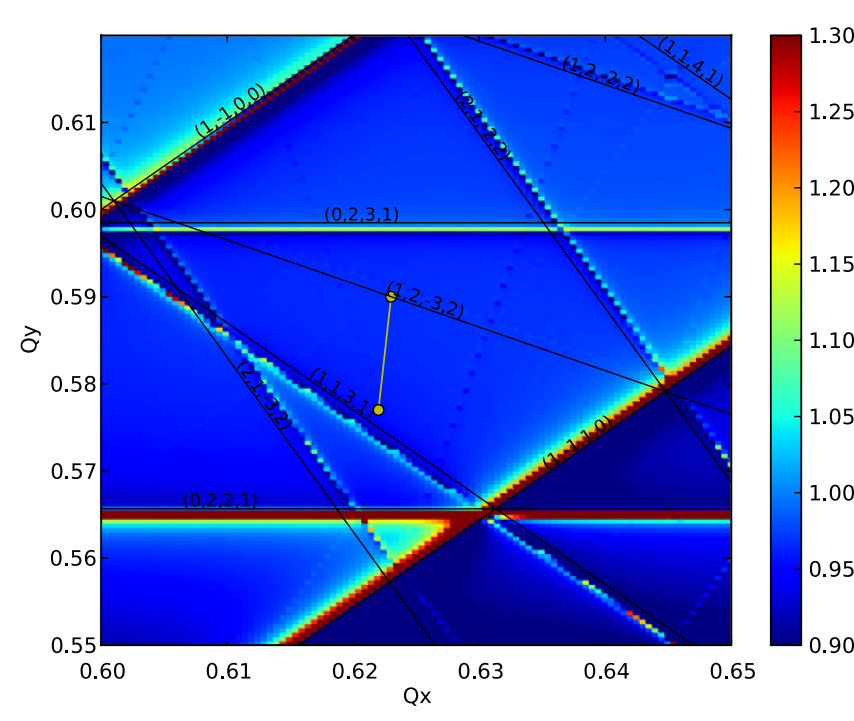

FIG. 7. Simulated tune scan based on a lattice model that includes magnet misalignments and corrector magnet settings determined according to our emittance tuning procedure. The yellow line shows how the coherent tunes increase as a bunch decays from $1.3 \times 10^{11}$ down to $1.6 \times 10^{9}$ particles.

seen in the simulated scan do not appear in the experimental scan. The working points for the IBS measurements are chosen with consideration of both the simulated and measured tune scans; further adjustments are often needed based on machine behavior.

\section{SIMULATION LATTICES}

An element-by-element description of CesrTA is used for the analytic and tracking calculations shown here. This description includes quadrupoles, sextupoles, bends, steerings, skew quadrupole correctors, wigglers, and rf cavities. Systematic multipoles are included for those sextupoles which have skew quadrupole or vertical steering windings. We use an analytic model of the damping wiggler field, which is based on a fit to a finite element calculation [26]. Tracking through wigglers is by symplectic integration.

The vertical IBS rise time depends on the dispersion. However, vertical dispersion is zero for an ideal flat ring. Vertical dispersion is included in our analytic IBS calculations by introducing $y z$ coupling into the 1 -turn transfer matrix. This is done at each element by augmenting the 1-turn transfer matrix before utilizing it in the analytic IBS calculation. The transfer matrix $\mathbf{T}$ is replaced with $\tilde{\mathbf{T}}$, where $\tilde{\mathbf{T}}=\mathbf{T W}$, and

$$
\mathbf{W}=\left(\begin{array}{cccccc}
1 & 0 & 0 & 0 & 0 & 0 \\
0 & 1 & 0 & 0 & 0 & 0 \\
0 & 0 & 1 & 0 & 0 & -\tilde{\eta}_{y} \\
0 & 0 & 0 & 1 & 0 & -\tilde{\eta}_{y}^{\prime} \\
0 & 0 & \tilde{\eta}_{y}^{\prime} & -\tilde{\eta}_{y} & 1 & 0 \\
0 & 0 & 0 & 0 & 0 & 1
\end{array}\right) .
$$

This transformation preserves the symplecticity of the transfer matrix. $\tilde{\eta}_{y}$ and $\tilde{\eta}_{y}^{\prime}$ are dispersionlike quantities. An ideal lattice modified according to the above prescription with $\tilde{\eta}_{y}=0.01 \mathrm{~m}$ and $\tilde{\eta}_{y}^{\prime}=0.002$ has an rms vertical dispersion of $10.9 \mathrm{~mm}$ and a vertical IBS rise time similar to that of a lattice with an rms vertical dispersion of $10 \mathrm{~mm}$.

The vertical dispersion in CesrTA is measured to be less than $15 \mathrm{~mm}$. The upper bound is limited by the resolution of our measurement technique. The coupling is determined by direct measurement to be $\bar{C}_{12}<0.003$, using an extended Edwards-Teng formalism [29]. This amount of coupling is negligible, and the simulation assumes no transverse coupling.

The analytic simulation takes the measured low current horizontal and vertical beam sizes and bunch length as input parameters and computes the current dependence. The horizontal emittance used in the calculation is chosen to match the measured near zero current emittance. The vertical emittance is also set to agree with the measurement extrapolated to zero current. (The vertical emittance of the design simulation lattice is zero.) The energy spread and bunch length used in the simulation are obtained by evaluating the standard radiation integrals.

The Monte Carlo simulation includes photon emission and so requires a realistic vertical dispersion function. This is generated by applying a distribution of misalignments to the ideal lattice, then correcting the phase advance, coupling, orbit, and vertical dispersion according to the same procedure that is applied to CesrTA low-emittance tuning [30]. The magnitude of the misalignments is set such that the zero current vertical emittance is roughly $15 \mathrm{pm} \mathrm{rad}$.

\section{EXPERIMENT}

For measurements of intrabeam scattering, we load a specific lattice configuration into the storage ring, which includes beam energy, working point, and rf voltage. The orbit, betatron phase, transverse coupling, and dispersion are measured at each of the 100 beam position monitors around the ring and then corrected to match the design. The phase and coupling data is derived from turn-by-turn position measurements at each of the beam position monitors for a resonantly excited beam [31]. The measurement of betatron phase and coupling takes 10 seconds, with phase reproducibility of order $0.05 \mathrm{deg}$ and $\bar{C}_{12}$ reproducibility of 0.002 . The dispersion is determined by directly measuring the dependence of closed orbit on beam energy ( $\mathrm{rf}$ frequency). The machine model is fit to the measured phase, coupling, and dispersion by varying all 100 quadrupoles, 25 skew quadrupole, and 55 vertical steering correctors. The machine optics are forced to match the design by loading the fitted magnet changes with opposite sign. The procedure typically converges in a few iterations. An example measurement of the optical functions after correction and just prior to an IBS run is shown in Fig. 8. The machine is tuned for minimum vertical emittance 

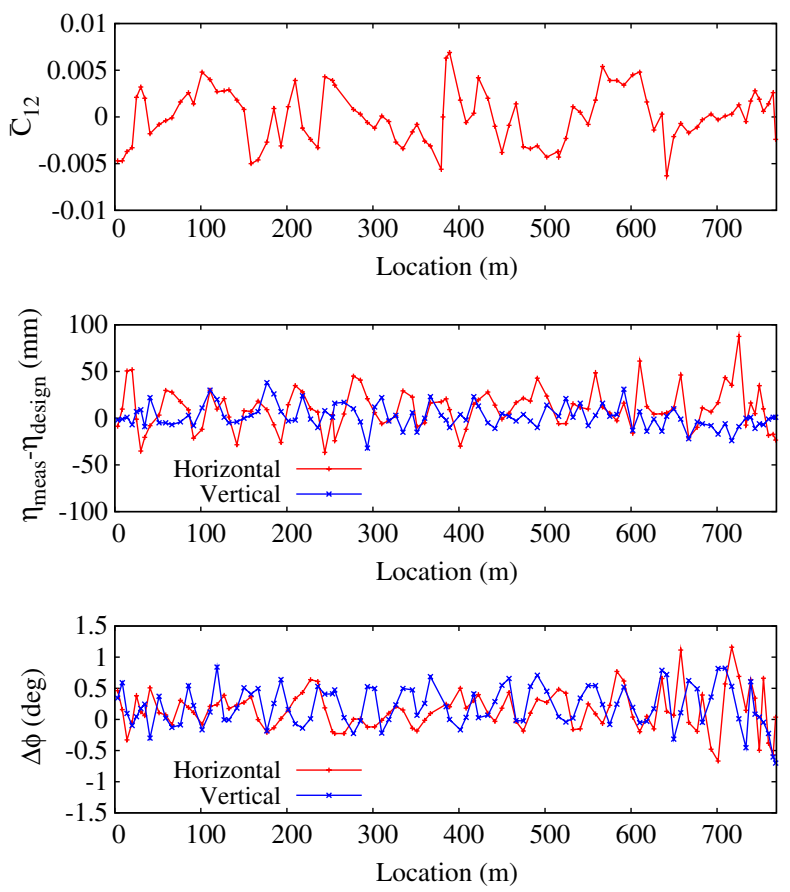

FIG. 8. Top: Measured transverse coupling at each beam position monitor after correction. $\bar{C}_{12}$ is the ratio of the normalized amplitude of vertical to horizontal motion when the beam is driven at the $a$-mode (horizontal) tune. Middle: Measured dispersion error with respect to design $\left(\eta_{\text {measured }}-\eta_{\text {design }}\right)$. Bottom: The residual betatron phase error $\left(\phi_{\text {measured }}^{h, v}-\phi_{\text {design }}^{h, v}\right)$. A $1^{\circ} \mathrm{rms}$ phase error corresponds to a $\sim 2 \% \beta$ error.

according to the algorithm given in [30]. For experiments requiring a larger beam size, the vertical emittance is increased by adjusting a closed coupling and vertical dispersion bump that propagates vertical dispersion through the wigglers.

A single bunch of about $1.6 \times 10^{11}$ particles $(10 \mathrm{~mA})$ is allowed to decay. The measurements include horizontal and vertical beam sizes, streak camera measurements of the longitudinal profile, and tunes in all three dimensions. The beam current decays from 10 to $1 \mathrm{~mA}$ in about 20 minutes. The short beam lifetime is due to Touschek scattering; below $1 \mathrm{~mA}$, where the charge density is considerably smaller, the beam lifetime improves significantly. In the interest of time, a large-amplitude pulsed orbit bump is used to scrape particles out of the beam in $0.25 \mathrm{~mA}$ decrements. The discontinuities in the data at bunch charge $<2 \times 10^{10}$ particles correspond to the regime where beam is scraped out.

IBS measurements are taken during dedicated periods of CesrTA operation. The IBS measurements in 2011 led us through iterative improvements in our understanding of how to operate the accelerator and how to measure IBS effects. Improvements on the accelerator side included a better understanding of the tunes and the selection of the working point (tunes as determined by lattice optics), a better understanding of the coupling and its impact on the measurements, and the development of more exact procedures for establishing the desired machine configuration. Improvements to the instrumentation included the implementation of beam size measurements for both electrons and positrons and the development of more accurate and robust analysis software. The data presented here were taken during the April 2012 CesrTA run. The measurements in December 2012 and April 2013 corroborate and expand on the April 2012 run and have been published in [32,33].

Figure 9 shows the data from a positron bunch in conditions tuned for minimum vertical emittance. Analytic
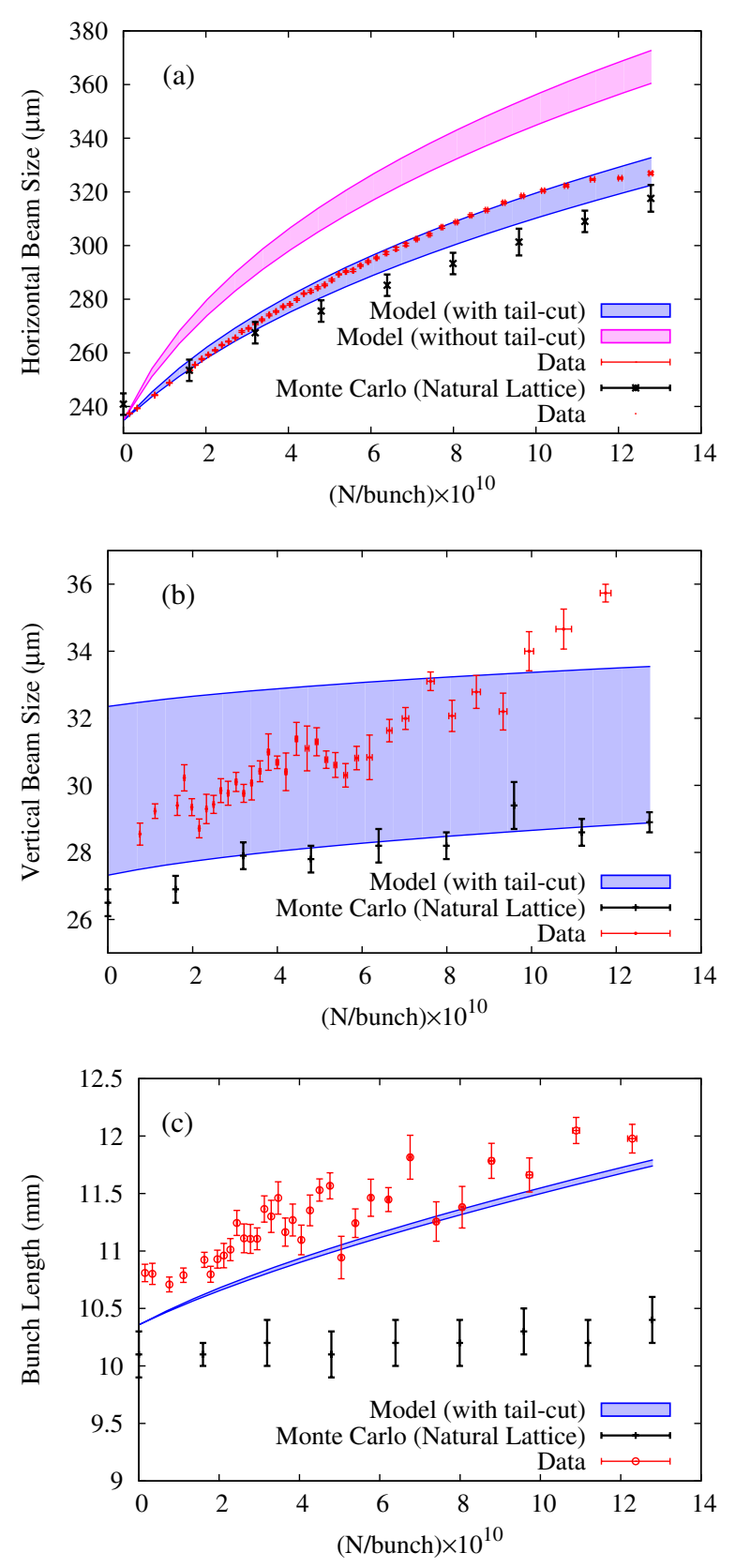

FIG. 9. (a) Horizontal, (b) vertical, and (c) longitudinal beam size versus current for $e^{+}$bunch in conditions tuned for minimum vertical emittance. 
TABLE III. Approximate statistical uncertainties at high current.

\begin{tabular}{lc}
\hline \hline Measurement & Uncertainty \\
\hline Current (horizontal binning) & $0.3 \%$ \\
Current (bunch length binning) & $0.9 \%$ \\
Horizontal size & $0.2 \%$ \\
Bunch length & $1.0 \%$ \\
Vertical size & $0.2 \%$ \\
\hline \hline
\end{tabular}

results from the $\boldsymbol{\Sigma}$-matrix formalism described in Sec. II and the Monte Carlo simulation described in Sec. II B are shown along with the data. Some of the error bars in Fig. 9 are below the resolution of the plot. The approximate statistical uncertainties at high current are shown in Table III.

The systematic uncertainty in the measured horizontal beam size is about $2 \%$, and is due to vibration of optical elements and horizontal beam motion. The systematic uncertainty in the vertical measurement is about $\pm 2 \mu \mathrm{m}$ and is dominated by our understanding of the $\mathrm{x}$-ray optics and detector.

The accuracy of the simulation is limited by the ambiguity of the Coulomb log and limited knowledge of the zero current vertical beam size of the machine. The simulation result shown here follows the usual convention for the tail cut of one event/damping time as the cutoff.

The $\boldsymbol{\Sigma}$-matrix IBS simulation is run twice, once with a zero current vertical emittance that extends to the bottom range of the measurement systematic uncertainty, and once that extends to the upper range of the measurement systematic uncertainty. The shaded region is the area between those two results. This serves two purposes. First, it reflects the systematic uncertainty in the vertical beam size measurements. Second, it gives the reader an idea of how the horizontal simulation result depends upon particle density as determined by the vertical beam size.

The zero current vertical emittances that bound the data in Fig. 9(b) are 17.9 and $25.1 \mathrm{pm}$. The shaded regions of 9(a) and 9(c) show how the horizontal and vertical simulation results change as the zero current vertical emittance is varied from the lower bound to the upper bound.

The measured zero current horizontal emittance, which is an input parameter to the simulation, is $3.8 \mathrm{~nm}$ rad. For

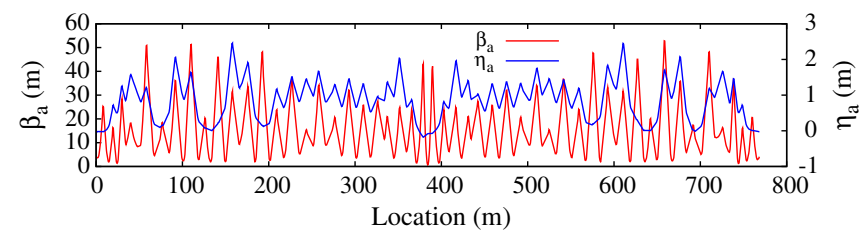

FIG. 10. CesrTA design $a$-mode (horizontal-like) $\beta$ and dispersion $\eta$. the bunch length and energy spread, we use the values calculated from the radiation integrals.

The simulation uses a perfectly aligned CesrTA lattice. Vertical dispersion is included by modifying the 1-turn transfer matrix with $\mathbf{W}$ before passing it to the IBS rise-time calculation. $\tilde{\eta}$ is set to $10 \mathrm{~mm}$.

The horizontal emittance increases from $3.8 \mathrm{~nm} \mathrm{rad}$ at low current $\left(<1.5 \times 10^{9}\right.$ particles/bunch) to $10.4 \mathrm{~nm} \mathrm{rad}$ at $1.3 \times 10^{11}$ particles/bunch. The reason for the relatively large horizontal blowup is the large horizontal dispersion in CesrTA. The lattice functions $\beta_{a}$ and $\eta_{a}$ are
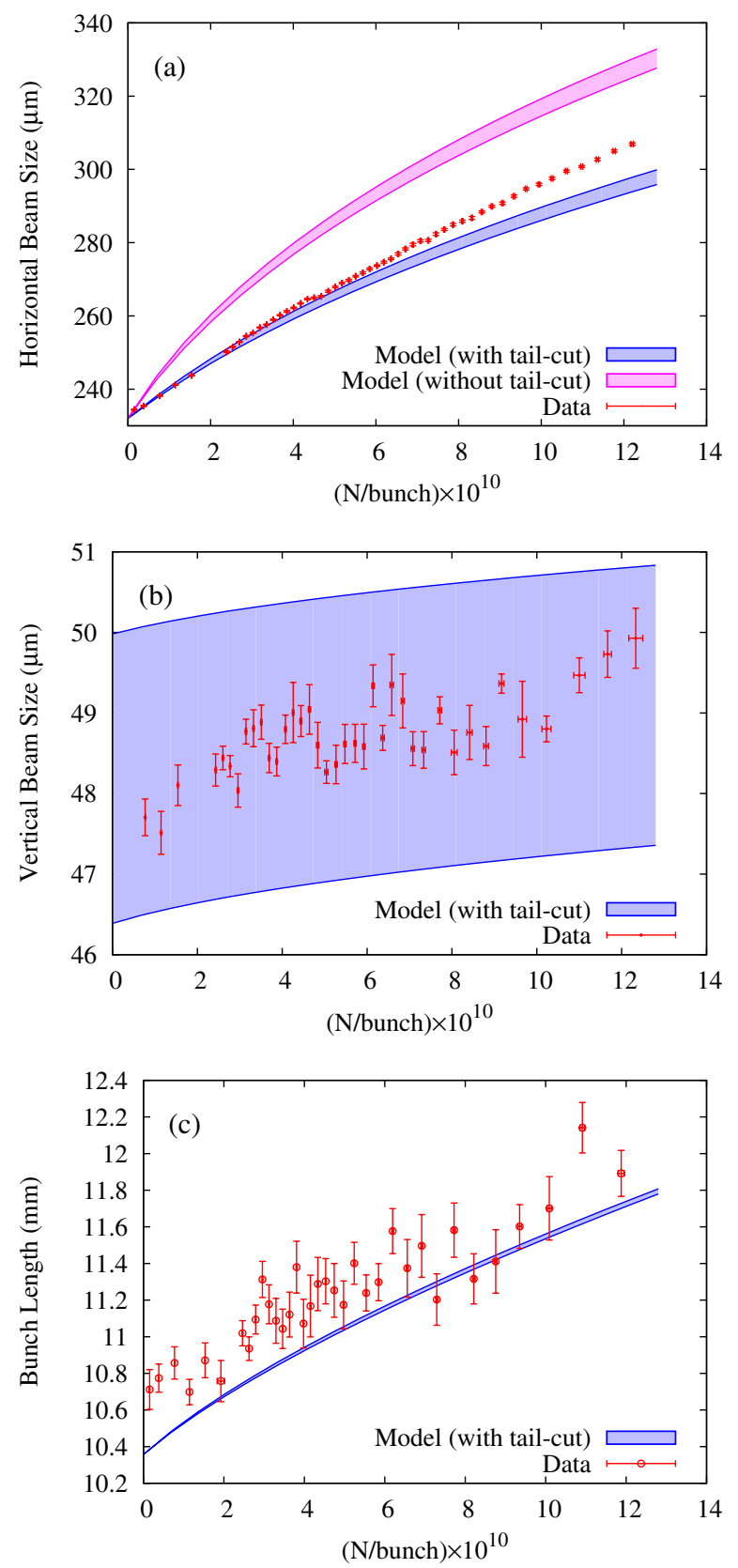

FIG. 11. (a) Horizontal, (b) vertical, and (c) longitudinal beam size versus current for $e^{+}$bunch with increased zero current vertical emittance. 
shown in Fig. 10. The rms horizontal dispersion, $\eta_{a}$, is $1.0 \mathrm{~m}$ and peaks at $2.5 \mathrm{~m}$. For comparison, the rms vertical dispersion is less than $15 \mathrm{~mm}$.

In Fig. 11 the zero current vertical emittance of the bunch was increased by propagating vertical dispersion through the damping wigglers with the help of a closed coupling and dispersion bump. The larger vertical beam size decreases the particle density, which in turn reduces the amount by which IBS blows up the horizontal beam size. The zero current horizontal emittance is $3.7 \mathrm{~nm} \mathrm{rad}$. The zero current vertical emittances that bound the data are 51.6 and $59.9 \mathrm{pm}$.
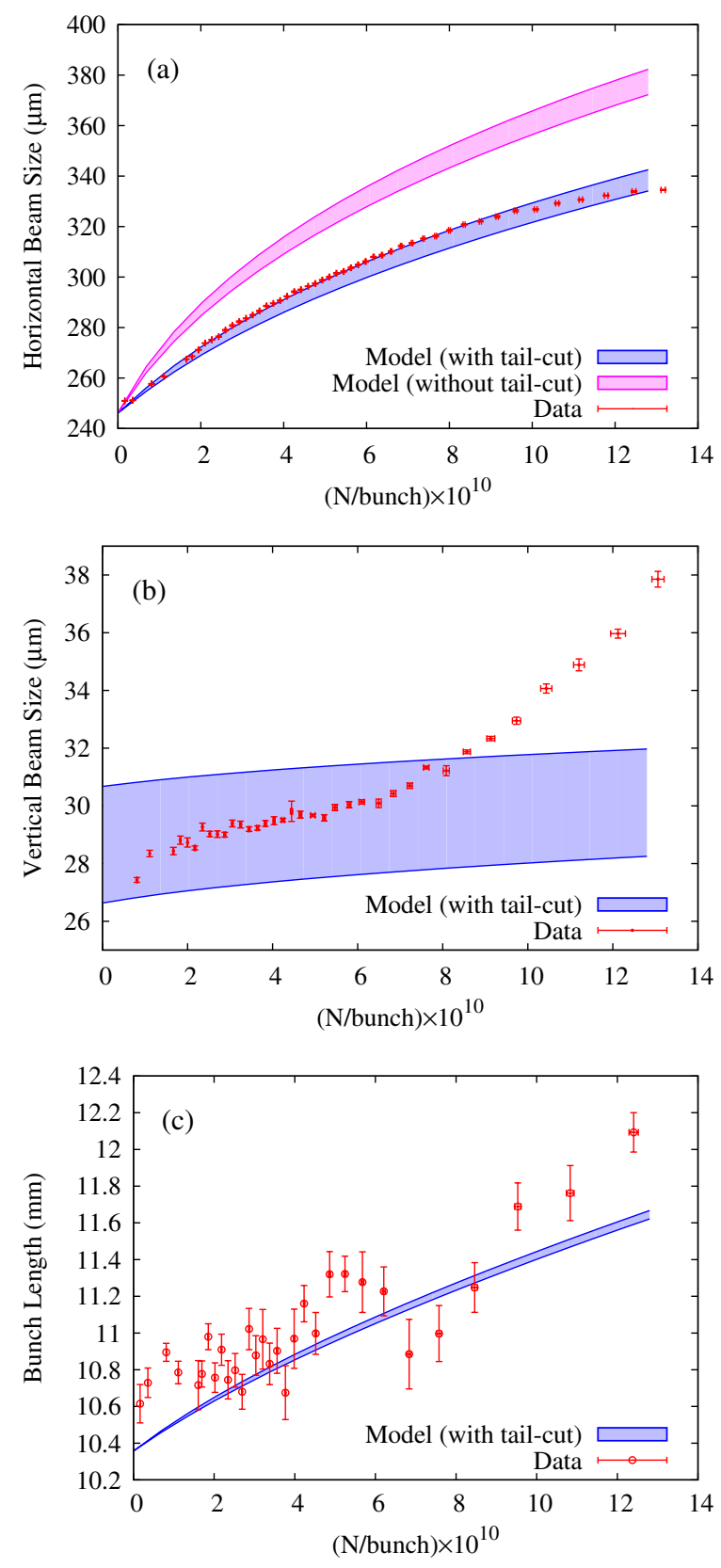

FIG. 12. (a) Horizontal, (b) vertical, and (c) longitudinal beam size versus current for $e^{-}$bunch in conditions tuned for minimum vertical emittance.
IBS theory is species independent. Measurements of both $e^{-}$and $e^{+}$can help identify machine and instrumentation systematics and distinguish IBS from species-dependent beam physics such as electron cloud and ion effects. Figure 12 shows data from an electron bunch in conditions tuned for minimum vertical emittance. The measured horizontal emittance is $4.3 \mathrm{~nm}$ rad at zero current and $8.2 \mathrm{~nm} \mathrm{rad}$ at $4.8 \times 10^{10}$ particles/bunch. The zero current vertical emittances that bound the data are 16.9 and $22.4 \mathrm{pm}$.

Data from an $e^{-}$run where the vertical emittance was increased are shown in Fig. 13. The horizontal emittance is
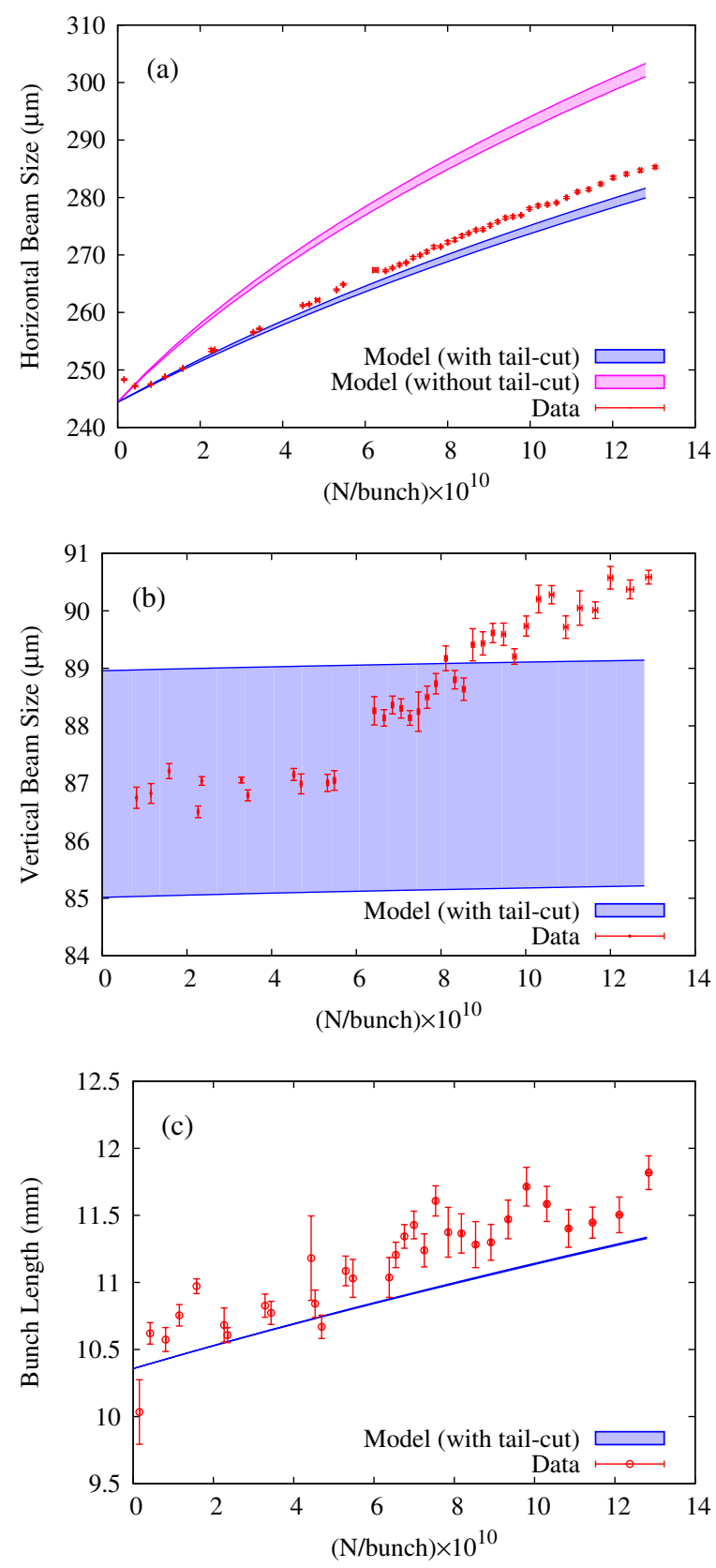

FIG. 13. (a) Horizontal, (b) vertical, and (c) longitudinal beam size versus current for $e^{-}$bunch with increased zero current vertical emittance. 

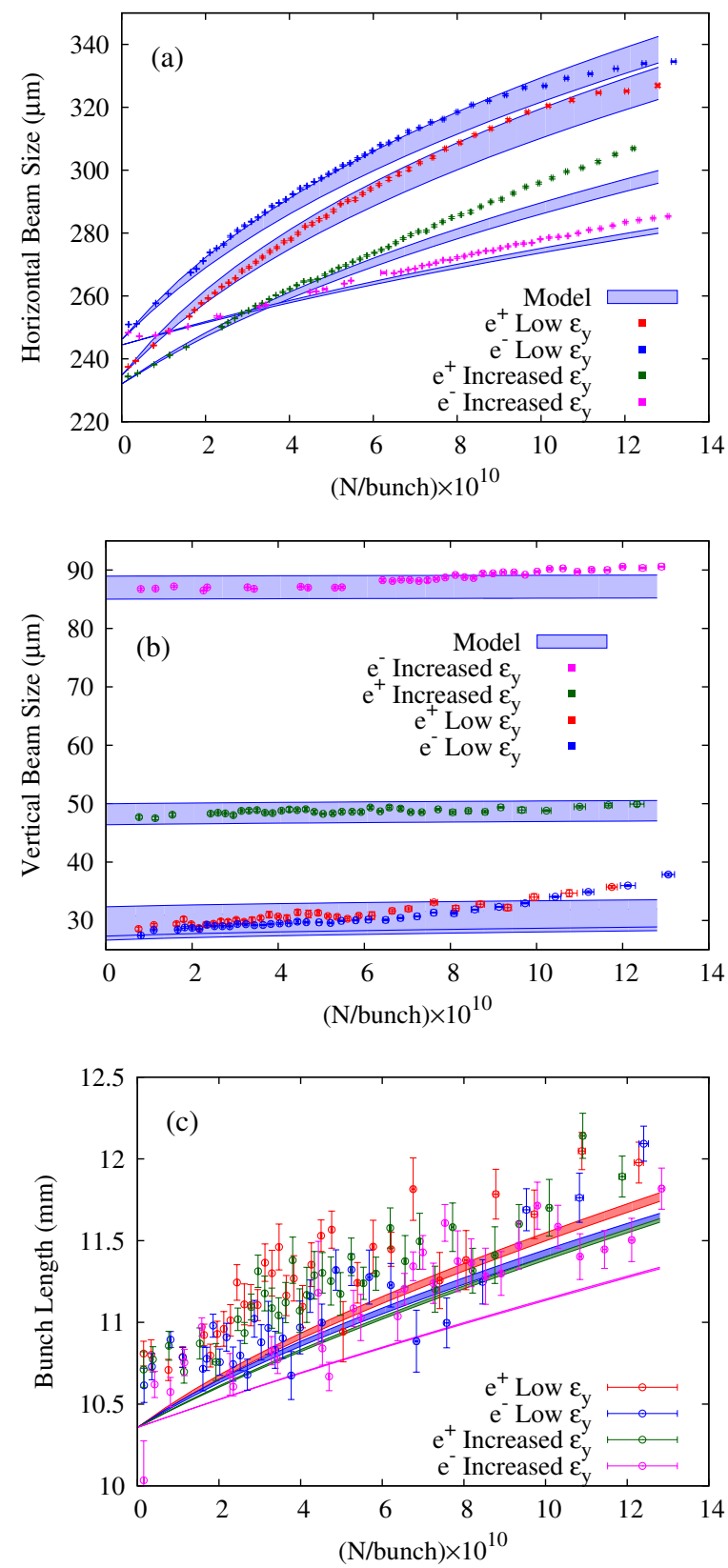

FIG. 14. Aggregated (a) horizontal, (b) vertical, and (c) longitudinal data comparing $e^{+}$and $e^{-}$in minimum emittance conditions and conditions where the zero current vertical emittance was blown up using closed coupling and dispersion bumps.

$4.2 \mathrm{~nm} \mathrm{rad}$ at zero current and $5.5 \mathrm{~nm} \mathrm{rad}$ at $4.8 \times$ $10^{10}$ particles/bunch. The vertical emittances that bound the data are 172 and $188 \mathrm{pm}$.

Figure 14 shows the combined data from the two $e^{-}$and two $e^{+}$data sets.

\section{DISCUSSION}

\section{A. Data}

IBS effects are most evident in the horizontal dimension, where large horizontal dispersion leads to significant blowup. In comparison, IBS is not a strong effect in the vertical. This is because the vertical dispersion is so small. The direct transfer of momentum from the horizontal to the vertical by IBS is small at high energy.

The amount of the blowup can be controlled by varying the vertical emittance, and thus the particle density. The simulations show bunch lengthening due to IBS, but we are unable to distinguish IBS lengthening from potential well distortion in our measurements.

An interesting anomaly we have encountered is the behavior of the vertical beam size at high currents. The effect is seen in Figs. 9(b) and 12(b) above $8 \times 10^{10}$ particles/bunch. We observe that vertical beam size plotted versus current increases with positive curvature. Much more severe cases of this blowup have been observed during the machine studies. We find that adjusting betatron and synchrotron tunes during experiments affects the blowup, but in a somewhat unpredictable way. The blowup is observed in both electron and positron beams.

The observed blowup in the vertical does not appear to be an instrumentation effect because when the vertical size blows up, the horizontal size drops. This is because the blowup in the vertical reduces the particle density, which reduces the IBS effect in the horizontal.
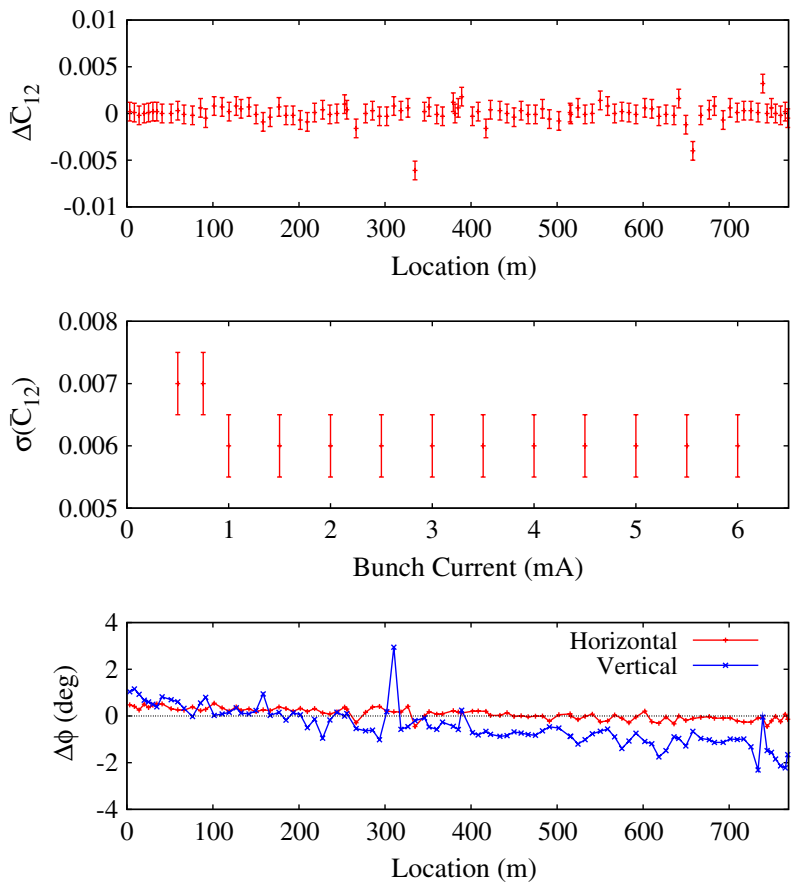

FIG. 15. Top: Difference in transverse coupling at $6 \mathrm{~mA}$ vs $0.8 \mathrm{~mA}$ single-bunch current measured at each BPM. Middle: Current dependence of rms $(\sigma)$ of residual coupling of all $N$ BPMs where $\sigma^{2}=\frac{1}{N} \sum_{i=1}^{N=100}\left(\bar{C}_{i 12}\right)^{2}$. Bottom: Difference in betatron phase at $6 \mathrm{~mA}$ vs $0.8 \mathrm{~mA}$ single-bunch current. The downward trend in the vertical corresponds to the currentdependent tune shift. $\beta$ is related to the phase advance from one BPM to the next. The fractional change in $\beta$ from 6 to $0.8 \mathrm{~mA}$ is less than $1 \%$. 
At high current, the vertical beam centroid position over 32768 turns was recorded using the xBSM. A fast Fourier transform of this data does not show a clear signal above background, so we cannot attribute the anomalous growth in vertical size to an instability. Adjustments to the corrected chromaticity did not impact the blowup.

Measurements of coupling $\bar{C}_{12}$ at different bunch currents are shown in Fig. 15. There is no evidence of significant current-dependent transverse coupling.

The measured bunch length shown here is consistently about $0.5 \mathrm{~mm}$ longer than the predicted value. Measurement results from December 2012 do not show this discrepancy. Between April and December 2012 the streak camera was realigned and the analysis software was improved. However, the evidence does not point to any particular instrumentation systematic.

\section{B. Theory}

The presence of the Coulomb log is a well-known ambiguity in IBS theory as it requires the introduction of loosely defined cutoffs in the minimum and maximum scattering angle. The choice of one event per damping time as the boundary between multiple-event and singleevent scattering is somewhat arbitrary. That said, the data shown here are in reasonable agreement with theory, suggesting that with implementation of the tail cut, the IBS theory is a reasonable model of performance for electron and positron machines. Furthermore, as shown in Fig. 2(b), the theory gives a good description of the data even when the large angle cutoff used in the calculation is varied by more than an order of magnitude.

The theory used here is Kubo and Oide's $\boldsymbol{\Sigma}$-matrix based IBS formalism. This model is a generalization of Bjorken and Mtingwa's formalism that can handle arbitrary coupling of the horizontal, vertical, and longitudinal motion. It includes the tail cut. Coupling in CesrTA for the experiments shown here was not large enough to noticeably impact the IBS growth rates. If coupling were significantly larger, then the predictions from Kubo and Oide's method may diverge from those of Bjorken and Mtingwa's method. Additional IBS studies with more strongly coupled beams are planned to investigate this regime.

\section{CONCLUSION}

We have presented data on intrabeam scattering in a high-intensity, wiggler-dominated, $e^{-} / e^{+}$storage ring. Additional current-dependent effects, such as tune shift and potential well distortion, have been observed in the beams. An anomalous blowup in the vertical dimension is seen at high current and requires further study.

This data has been compared to a generalized version of the Bjorken-Mtingwa formalism for calculating IBS effects. The results presented here suggest that, provided the tail-cut procedure is applied, existing IBS theory is an accurate predictor of machine performance in $e^{+} / e^{-}$machines.
Further IBS studies at CesrTA include adjusting wiggler parameters to observe how the equilibrium emittance of an IBS-dominated beam depends on the damping time, measurements at 1.8, 2.3, and $2.5 \mathrm{GeV}$, and measurements with different rf voltages. There are also plans to explore IBS in strongly coupled beams. Some of these studies have been completed and are documented in [21,22].

\section{ACKNOWLEDGMENTS}

The authors would like to thank Mike Billing for his many helpful discussions. We would like to thank Avi Chatterjee for his assistance during the December 2012 experiments. The experiments reported here would not have been possible without the diligent support of the CESR Operations Group. This research was supported by NSF and DOE Contracts No. PHY-0734867, No. PHY-1002467, No. PHYS-1068662, No. DE-FC02-08ER41538, No. DE-SC0006505, and the Japan/U.S. Cooperation Program.

[1] B. Barish, M. Harrison, K. Yokoya, and B. Foster, Global Design Effort Technical Report 2013.

[2] Y. Papaphilippou, in Proceedings of the 3rd International Particle Accelerator Conference, New Orleans, Louisiana, 2012 (IEEE, Piscataway, NJ, 2012), pp. 1368-1370.

[3] Y. Cai, K. Bane, R. Hettel, Y. Nosochkov, M. H. Wang, and M. Borland, Phys. Rev. ST Accel. Beams 15, 054002 (2012).

[4] V.N. Litvinenko, S. Belomestnykh, I. Ben-Zvi, M. M. Blaskiewicz, K. A. Brown, J. C. Brutus, A. Elizarov, and A. Fedotov, ICFA Beam Dyn. Newslett. 58, 52 (2012).

[5] K. Kubo, S. K. Mtingwa, and A. Wolski, Phys. Rev. ST Accel. Beams 8, 081001 (2005).

[6] V. Lebedev, in High Intensity and High Brightness Hadron Beams: 33rd ICFA Advanced Beam Dynamics Workshop, AIP Conf. Proc. No. 773, edited by I. Hoffman, J. M. Lagniel, and R.W. Hasse (AIP, New York, 2005), pp. 440-442.

[7] M. Martini, CERN Technical Report No. CERN PS/84-9, 1984.

[8] A. Piwinski, in Frontiers of Particle Beams, edited by M. Month and S. Turner (Springer, New York, 1988), pp. 297-309.

[9] A. V. Fedotov, W. Fischer, S. Tepikian, and J. Wei, in Proceedings of Hadron Beams 2006 (ICFA, Tsukuba, Japan, 2006), pp. 259-261.

[10] A. V. Fedotov et al., in Proceedings of Hadron Beams 2008 (ICFA, Nashville, Tennessee, 2008), pp. 148-152.

[11] K. L. F. Bane, H. Hayano, K. Kubo, T. Naito, T. Okugi, and J. Urakawa, Phys. Rev. ST Accel. Beams 5, 084403 (2002).

[12] M. Palmer et al., in Proceedings of the 23rd Particle Accelerator Conference, Vancouver, Canada, 2009 (IEEE, Piscataway, NJ, 2009), pp. 4200-4204.

[13] D. Sagan, Nucl. Instrum. Methods Phys. Res., Sect. A 558, 356 (2006).

[14] J. Shanks, D. Rubin, and D. Sagan (to be published). 
[15] J. P. Alexander and D. P. Peterson, in The Handbook of Accelerator Physics and Engineering, edited by A.W. Chao, K. H. Mess, M. Tigner, and F. Zimmerman (World Scientific, Singapore, 2013), p. 721, 2nd ed.

[16] N. T. Rider, M. G. Billing, M.P. Ehrlichman, D.P. Peterson, D. Rubin, J.P. Shanks, K. G. Sonnad, M. A. Palmer, and J.W. Flanagan, in Proceedings of the International Beam Instrumentation Conference 2012 (Tsukuba, 2012), pp. 585-589 [http://ibic12.kek.jp/ prepress/index.htm].

[17] S. T. Wang, D. Rubin, J. Conway, M. Palmer, D. Hartill, R. Campbell, and R. Holtzapple, Nucl. Instrum. Methods Phys. Res., Sect. A 703, 80 (2013).

[18] R. Holtzapple, M. Billing, D. Hartill, M. Stedinger, and B. Podobedov, Phys. Rev. ST Accel. Beams 3, 034401 (2000).

[19] K. Kubo and K. Oide, Phys. Rev. ST Accel. Beams 4, 124401 (2001).

[20] J.D. Bjorken and S. K. Mtingwa, Part. Accel. 13, 115 (1983).

[21] M. Ehrlichman, W. Hartung, M. Palmer, D. Peterson, D. Rider, N. Rubin, J. Shanks, C. Strohman, S. T. Wang, R. Campbell, R. Holtzapple, F. Antoniou, and Y. Papaphilippou, in Proceedings of the 3rd International Particle Accelerator Conference, New Orleans, Louisiana, 2012 (Ref. [2]), pp. 2970-2972.

[22] M. Ehrlichman, Ph.D. thesis, Cornell University, 2013.

[23] K. Kubo, in Proceedings of the 1st Strategic Accelerator Design Workshop (KEK, Tokyo, 1999), pp. 125-126.
[24] T. Raubenheimer, Part. Accel. 45, 111 (1994).

[25] T. Takizuka and H. Abe, J. Comput. Phys. 25, 205 (1977).

[26] D. Sagan, J. A. Crittenden, D. Rubin, and E. Forest, in Proceedings of the 20th Particle Accelerator Conference, Portland, OR, 2003 (IEEE, New York, 2003), pp. 1023-1025.

[27] M. Billing, Cornell University Technical Report No. CBN 80-02, 1980.

[28] A. Piwinski, in Proceedings of the 9th International Conference on High Energy Accelerators (NTIS, Springfield, VA, 1974), pp. 405-409.

[29] D. Sagan and D. Rubin, Phys. Rev. ST Accel. Beams 2, 074001 (1999).

[30] J. Shanks, in Proceedings of the 2011 Particle Accelerator Conference, NY (IEEE, New York, 2011), pp. 1540-1542.

[31] D. Sagan, R. Meller, R. Littauer, and D. Rubin, Phys. Rev. ST Accel. Beams 3, 092801 (2000).

[32] M. Ehrlichman, A. Chatterjee, W. Hartung, D. P. Peterson, N. Rider, D. Rubin, D. Sagan, J.P. Shanks, and S. T. Wang, in Proceedings of the International Particle Accelerator Conference 2013 (JACoW, Geneva, 2013), pp. 1126-1128.

[33] M. Ehrlichman, A. Chatterjee, W. Hartung, D. P. Peterson, N. Rider, D. Rubin, J.P. Shanks, and S. T. Wang, in Proceedings of the International Particle Accelerator Conference 2013 (JACoW, Geneva, 2013), pp. 1715-1717. 